\title{
LHomme
}

L'HOMME Revue française d'anthropologie

$193 \mid 2010$

Claude Lévi-Strauss (1908-2009)

\section{La vision du monde de Claude Lévi-Strauss}

\section{Emmanuel Terray}

\section{OpenEdition}

Journals

Édition électronique

URL : http://journals.openedition.org/lhomme/24346

DOI : 10.4000//homme.24346

ISSN : 1953-8103

\section{Éditeur}

Éditions de l'EHESS

\section{Édition imprimée}

Date de publication : 19 février 2010

Pagination : 23-44

ISSN : 0439-4216

\section{Référence électronique}

Emmanuel Terray, "La vision du monde de Claude Lévi-Strauss », L'Homme [En ligne], 193 | 2010, mis en ligne le 29 janvier 2012, consulté le 21 décembre 2020. URL : http://journals.openedition.org/ Ihomme/24346 ; DOI : https://doi.org/10.4000//homme.24346 


\title{
La vision du monde de Claude Lévi-Strauss
}

\section{Editions de l'E.H.E.S.S. | L'Homme}

\author{
$2010 / 1-n^{\circ} 193$ \\ pages 23 à 44 \\ ISSN 0439-4216
}

Article disponible en ligne à l'adresse:

http://www.cairn.info/revue-I-homme-2010-1-page-23.htm

Pour citer cet article :

"La vision du monde de Claude Lévi-Strauss", L'Homme, 2010/1 n 193, p. 23-44.

Distribution électronique Cairn.info pour Editions de l'E.H.E.S.S..

(C) Editions de l'E.H.E.S.S.. Tous droits réservés pour tous pays.

La reproduction ou représentation de cet article, notamment par photocopie, n'est autorisée que dans les limites des conditions générales d'utilisation du site ou, le cas échéant, des conditions générales de la licence souscrite par votre établissement. Toute autre reproduction ou représentation, en tout ou partie, sous quelque forme et de quelque manière que ce soit, est interdite sauf accord préalable et écrit de l'éditeur, en dehors des cas prévus par la législation en vigueur en France. II est précisé que son stockage dans une base de données est également interdit. 


\title{
La vision du monde de Claude Lévi-Strauss
}

\author{
Emmanuel Terray
}

\begin{abstract}
$\grave{A}$
CÔTÉ DE SES CEUVRES proprement scientifiques consacrées à la parenté et aux mythes, Claude Lévi-Strauss nous propose non pas une philosophie - il a plusieurs fois vigoureusement récusé le terme - mais une vision du monde accompagnée d'une vision de l'histoire du monde. Celles-ci procèdent de ses recherches, mais elles ne s'en déduisent pas, du moins pas entièrement; elles contiennent des éléments originaux qui appellent de notre part une attention particulière.
\end{abstract}

La vision du monde de Lévi-Strauss découle, à mon avis, de deux propositions qui ont en quelque sorte valeur d'axiomes. Première proposition : l'être humain est partie intégrante de la nature, et il ne saurait en être dissocié que de façon artificielle et illusoire. Deuxième proposition: la diversité des cultures est à l'origine de toute création et de tout progrès ; bien plus, dira Lévi-Strauss dans Myth and Meaning, "je ne vois pas comment le genre humain pourrait réellement vivre sans quelque diversité interne» (1978: 16). Examinons ces deux propositions tour à tour.

Lêtre humain est partie intégrante de la nature. Tout d'abord, il est un être vivant, il a un corps, un organisme, et comme tel il est soumis aux lois de la biologie. La nature le nourrit; elle peut aussi le rendre malade; elle gouverne le cours de sa vie, de la naissance et de l'enfance à la vieillesse et à la mort. Mais Lévi-Strauss va beaucoup plus loin que ce constat banal. À ses yeux, l'esprit lui-même et la pensée, au moyen desquels on creuse d'ordinaire un abîme entre l'être humain et la nature, sont en fait partie intégrante de celle-ci : "L'esprit aussi est une chose", lisons-nous dans La Pensée sauvage (1962: 328, note). Dans Tristes Tropiques, Lévi-Strauss écrivait déjà : "Ma pensée est elle-même un objet. Étant "de ce monde", 
elle participe de la même nature que lui» (1955: 47). Il reprendra le même propos dans L'Homme $n u$ : «La pensée et le monde qui l'englobe et qu'elle englobe sont deux manifestations corrélatives d'une même réalité » (1971: 605).

C'est précisément parce que l'être humain et son esprit font partie de la nature qu'ils peuvent la penser et la connaitre : «L'esprit ne peut comprendre le monde que parce qu'il est un produit et une partie de ce monde", est-il affirmé dans Le Regard éloigné (1983: 163). La portée de ces formules est considérable : si on les prend au sérieux, c’est l'antique et célèbre "problème de la connaissance " qui se dissout tout entier et d'un seul coup. On se rappelle comment ce problème était posé: comment l'esprit et la pensée peuvent-ils comprendre et connaître le monde? Mais l'énoncé même de la question présupposait que l'esprit et le monde fussent deux réalités distinctes, différentes par nature, et dont il fallait par conséquent analyser les rapports et le rapprochement éventuel. Entre les deux termes se glissait bien la sensibilité ou la perception, mais on la plaçait d'ordinaire du côté du monde, et le problème devenait alors celui des relations entre la sensibilité et l'entendement. À vrai dire, dès le XVIII ${ }^{\mathrm{e}}$ siècle, le philosophe allemand Johann Georg Hamann, examinant la doctrine de son "ami » Kant, avait mis en cause l'opposition tranchée instituée par ce dernier entre les deux facultés :

«La sensibilité et l'entendement, les deux branches de la connaissance humaine, ne proviennent-elles pas d'une racine commune, mais qui nous est inconnue, si bien que les objets nous sont donnés par l'une et pensés (compris et conceptualisés) par l'autre? À quoi bon une séparation si violente et illicite de ce que la nature a assemblé ? Les deux branches ne vont-elles pas dépérir et dessécher à cause de cette dichotomie ou division de leur racine transcendantale?» (2001: 146).

Lévi-Strauss va s'engager résolument dans la voie suggérée par Hamann. Si l'entendement, étudiant la parenté ou les mythes, met au jour des structures, c'est parce qu'il travaille sur des données qui sont déjà structurées par la sensibilité : comme il est dit dans L'Homme nu, "aucun caractère n'est marquant en soi, et c'est l'analyse perceptive, déjà combinatoire et capable d'une activité logique au niveau de la sensibilité, qui, relayée par l'entendement, confere une signification aux phénomènes et les érige en texte» (1971: 501). Mais la matière fournie par la sensibilité est structurée parce que le monde est lui-même structuré :

"Quand l'esprit se saisit de données empiriques préalablement traitées par les organes des sens, il continue à travailler structuralement, pour ainsi dire, une matière qu'il reçoit déjà structurée. Il ne pourrait le faire si l'esprit, le corps auquel l'esprit appartient, et les choses que le corps et l'esprit perçoivent n'étaient partie intégrante d'une seule et même réalité" (Lévi-Strauss 1983: 163). 
L'esprit, le corps, les choses : voici qu'une continuité rigoureuse est rétablie entre ces trois termes, fondée sur une parfaite communauté de nature. On a parlé de kantisme à propos de Lévi-Strauss, et lui-même n’a pas écarté cette imputation, à condition de préciser qu'il s'agit d'un kantisme sans sujet transcendantal. Pour ma part, je parlerais plus volontiers de monisme, de ce monisme implicite qui est en quelque sorte présupposé par l'existence même de l'activité scientifique.

Seconde proposition : la diversité des cultures est nécessaire à l'existence même du genre humain, et elle est en quelque sorte consubstantielle à la nature humaine. Partons, comme le fait Lévi-Strauss lui-même, de la définition classique de la culture proposée par Edward B. Tylor : la culture est " ce tout complexe qui comprend les savoirs, les croyances, l'art, la morale, la coutume et toute capacité ou habitude acquise par l'homme en tant que membre de la société » (1958: 1). À cette définition, Lévi-Strauss (1983: 39) ajoutera simplement que les éléments constitutifs d'une culture ne sont pas un agrégat sans cohésion résultant du jeu des circonstances : ils forment système; leur association bénéficie ainsi d'une relative stabilité dans le temps. La diversité des cultures est tout d'abord un fait, constaté, au moins depuis Hérodote et Ibn Khaldoun, par l'histoire comme par la géographie et l'ethnologie. Mais sa reconnaissance pose aussitôt un problème : comment penser à la fois l'unité du genre humain et la diversité des cultures? Même si elle a été parfois contestée par certains extrémistes, la thèse de l'unité du genre humain a toujours fini par s'imposer à la conscience collective, et elle est aujourd'hui confirmée par la science. Dès lors, comment faire le partage de ce qui est propre à l'être humain en tant qu'être générique et de ce qui relève de la diversité des cultures? L'héritage commun ne saurait se réduire au corps et aux aspects biologiques de l'existence, car autrement, il n'y aurait pas de communication possible entre les cultures; or nous savons bien qu'entre elles, les emprunts, les échanges, les brassages se sont multipliés. Il y a donc un socle culturel commun, ou encore des universaux culturels, qui assurent l'unité de l'espèce sur le plan même de la culture. En quoi consistent ces universaux? De nombreuses listes ont été proposées; au XVIII ${ }^{\mathrm{e}}$ siècle, par exemple, Vico énumérait la religion, le mariage et les funérailles. On sait par ailleurs le rôle que LéviStrauss fait jouer à la prohibition de l'inceste : en tant que règle, il s'agit d'un fait de culture, mais son caractère universel la rapproche de la nature, et dans Les Structures élémentaires de la parenté, elle sera posée comme l'opérateur du passage de la nature à la culture.

Mais quels que soient le nombre et la nature de ces universaux, ils ne restreignent pas l'espace occupé par la diversité : il s'agit en quelque sorte d'un ensemble de cases vides qu'il appartient à la diversité de remplir. 
Pour user d'une autre métaphore, les universaux délimitent un certain nombre de terrains vierges sur lesquels la diversité vient s'épanouir et prospérer. Il en est ainsi de la prohibition de l'inceste : seul son principe est universel ; comme Lévi-Strauss lui-même l'a établi, elle prend des formes infiniment variées d'une société à l'autre et d'une culture à l'autre.

Quant à la vertu créatrice de la diversité des cultures, elle est clairement affirmée dans Myth and Meaning: "Les différences sont extrêmement fécondes. C'est seulement à travers la différence que le progrès s'est accompli » (1978 : 16). Le petit livre intitulé Race et Histoire est tout entier consacré à l'explicitation de cette thèse. Partant de l'exemple du jeu, LéviStrauss montre que les chances d'une coalition de parieurs sont beaucoup plus grandes que celles d'un joueur isolé pour obtenir un résultat déterminé, et il poursuit :

"Or cette situation ressemble beaucoup à celle des cultures qui sont parvenues à réaliser les formes d'histoire les plus cumulatives. Ces formes extrêmes n'ont jamais été le fait de cultures isolées, mais bien de cultures combinant volontairement ou involontairement leurs jeux respectifs, et réalisant par des moyens variés (migrations, emprunts, échanges commerciaux, guerres) ces coalitions dont nous venons d'imaginer le modèle [...]. Mais aucune culture n'est seule, elle est toujours donnée en coalition avec d'autres cultures, et c'est cela qui permet d'édifier des séries cumulatives [...]. La chance qu'a une culture de totaliser cet ensemble complexe d'inventions de tous ordres que nous appelons une civilisation est fonction du nombre et de la diversité des cultures avec lesquelles elle participe à l'élaboration - le plus souvent involontaire d'une commune stratégie" (in 1973: 413-414).

Et Lévi-Strauss de conclure magnifiquement: "L'exclusive fatalité, l'unique tare qui puissent affliger un groupe humain et l'empêcher de réaliser pleinement sa nature, c'est d'être seul » (in ibid. : 415).

Enfin, quelle relation peut-on concevoir entre les deux axiomes posés par Lévi-Strauss, appartenance à la nature et diversité des cultures? Dans le passé, deux hypothèses différentes ont été proposées pour rendre compte de celle-ci (cf. Lévi-Strauss 1983 : 22).

Première hypothèse : le genre humain aurait été dès l'origine scindé en plusieurs sous-espèces; c'est bien dans la nature - en l'occurrence celle de l'espèce humaine - que s'enracinerait la diversité. Mais, aux yeux de LéviStrauss, la pluralité des sous-espèces est une pure conjecture qui ne pourra jamais être vérifiée, car les origines humaines se perdent dans la nuit des temps ; quant aux rameaux dont on connaît l'existence, ils ont disparu, et il est vain de spéculer sur leur statut.

Seconde hypothèse : nous aurions affaire à une lignée initiale unique dont les ramifications se diversifieraient au fur et à mesure qu'elles se seraient installées dans des milieux différents. Mais de nouveau c'est bien la nature - à présent la variété des milieux naturels et des habitats - qui 
aurait engendré la diversité des cultures. Prévenons toute équivoque et tout malentendu: il n'y a, dans ce propos, aucun déterminisme géographique univoque; bien entendu, les êtres humains transforment les milieux qu'ils occupent, mais dans le cours même de cette activité s'établit une relation spécifique, donnant lieu à une expérience particulière, et c'est la diversité des expériences ainsi réalisées qui conduit à la diversité des cultures.

Or, pendant une longue période de son histoire, le genre humain s'est conformé aux deux axiomes de Lévi-Strauss : il a respecté la nature dont il s'éprouvait partie intégrante, et il a maintenu la diversité des cultures.

Respect de la nature: Lévi-Strauss (1973 : 374) en fait l'une des trois caractéristiques majeures qui amènent les sociétés dites primitives à résister au développement économique tel que la civilisation industrielle le conçoit et l'impose. Ce respect se manifeste d'abord dans la pratique: même après la "révolution néolithique » - l'invention de l'agriculture, de la poterie, de la domestication des animaux - les êtres humains n'altèrent que très superficiellement le milieu naturel qu'ils occupent. Ils n'utilisent qu'une faible quantité d'énergie - force musculaire, énergie animale, puissance du feu et parfois de l'eau - et, pour travailler la terre, ils recourent aux méthodes de l'agriculture itinérante sur brûlis.

Bien entendu, ce qui est vrai des agriculteurs est vrai a fortiori des chasseurs-collecteurs. Il faut rappeler ici le célèbre article que Marshall Sahlins (1968) a consacré à "la première société d'abondance": à partir de données recueillies principalement chez les Bushmen de l'Afrique du Sud, Sahlins montre que ceux-ci ne consacrent à la recherche de leur subsistance qu'un nombre d'heures limité dans la journée, parce qu'ils ne se soucient pas d'accumuler des provisions et des réserves dont le poids et l'encombrement entraveraient leur mobilité. Du coup, comme LéviStrauss le constate pour sa part en Amazonie, "les tribus indigènes ont besoin d'énormes surfaces pour subsister; mais ici une surabondance de vie animale attestait que depuis des décades l'homme avait été impuissant à troubler l'ordre naturel » (1955: 353).

L'emprise des agriculteurs sur le milieu est évidemment plus forte, mais eux non plus ne s'intéressent guère à l'accumulation et sacrifient la productivité à la sécurité : il s'agit pour eux de garantir le renouvellement des sols contre tout risque d'épuisement, et de se protéger des aléas du climat en mettant en valeur des parcelles de qualité très variée. Leur objectif est d'assurer ce que Marx appelle leur "reproduction simple ", à l'identique; ils "refusent", ou il ne leur vient pas à l'idée, de se lancer dans l'aventure de la reproduction élargie avec tous les risques qu'elle implique. Du coup, ici encore le milieu n'est transformé qu'en surface. 
Ces caractéristiques placent les sociétés dites primitives sous l'étroite dépendance de la nature; elles en ont conscience, et cette dépendance se traduit dans leur idéologie. Tout d'abord, les êtres humains se considèrent non pas comme les propriétaires, mais au contraire comme les hôtes ou les locataires de la nature; et ils lui manifestent leur gratitude et leur allégeance à travers toutes sortes de rituels et à travers des institutions comme le " maître africain de la terre ». Corrélativement, la nature ne se réduit pas à la glèbe - plus ou moins - nourricière ; elle est surtout, rappelle LéviStrauss, « le terrain sur lequel l'homme peut espérer entrer en contact avec les ancêtres, les esprits et les dieux. Il y a donc dans la notion de nature une composante "surnaturelle" » (1973 : 374). Pour éclairer la différence qui sépare dans ce domaine les sociétés dites primitives des nôtres, Lévi-Strauss prend l'exemple de ce qu'il est convenu d'appeler «les bonnes manières "; présentes de part et d'autre, elles revêtent cependant ici et là une signification diamétralement opposée: "Au lieu, comme nous le pensons, de protéger la pureté interne du sujet contre l'impureté externe des êtres et des choses, les bonnes manières servent chez les sauvages à protéger la pureté des êtres et des choses contre l'impureté du sujet » (1968: 419).

La nature représente donc pour le "sauvage " une puissance devant laquelle il faut d'abord s'incliner avant de négocier avec elle. Mais la dépendance des êtres humains vis-à-vis de la nature ne se situe pas seulement sur le plan de la biologie ou de la physiologie ; elle est aussi d'ordre intellectuel. Ici se situe l'un des apports les plus précieux de l'œuvre de Lévi-Strauss; à partir d'une connaissance extraordinairement fine et précise de la flore et de la faune, les sociétés dites primitives élaborent une "logique du sensible", ou une "logique du concret", qui transforme les qualités sensibles - le haut, le bas, le chaud, le froid, la droite, la gauche, les couleurs, les saveurs, les odeurs - en autant de catégories permettant de classer les réalités qui nous entourent, d'établir entre elles des analogies ou des oppositions, bref de penser l'univers et d'en comprendre le cours (Lévi-Strauss 1973 : 83 ; 1988 : 155). Bien entendu, les qualités sensibles sont ici des instruments au service de l'entendement: il s'agit, dit LéviStrauss (1988: 155), de dépasser l'opposition entre le sensible et l'intelligible, ou encore d'intégrer le sensible au rationnel sans rien perdre de ses propriétés (1955 : 50). Ici, ce sont toutes les analyses du Totémisme aujourd'hui et de La Pensée sauvage qu'il faudrait reprendre; faute de place, nous renvoyons nos lecteurs à ces ouvrages. Ce sont aussi les Mythologiques, puisque, déclare Lévi-Strauss, « les mythes sont construits sur la base d'une logique des qualités sensibles qui ne fait pas de distinction tranchée entre les états de la subjectivité et les propriétés du cosmos » (1964: 246). Cette dernière indication est capitale : l'être humain, avons-nous dit, appartient 
de part en part à la nature; eh bien, c'est précisément la logique des qualités sensibles qui intervient comme intermédiaire entre les deux termes et qui assure leur union en effaçant les discontinuités susceptibles de les séparer.

L'appartenance à la nature et le respect qui lui est porté s'expriment enfin, dans les mythes, par une étrange nostalgie; les mythes en effet renvoient à un âge disparu où les animaux parlaient et où les métamorphoses - c'està-dire les passages entre le règne humain et les règnes animal et végétal se rencontraient couramment dans la vie de tous les jours (Lévi-Strauss 1967 : 59). En d'autres termes, le temps mythique est une ère pendant laquelle la fusion entre l'homme et la nature a été plus intime qu'elle ne le sera jamais par la suite, et beaucoup de sociétés primitives vivent l'effacement de cet âge d'or comme la première étape du déclin du genre humain.

Tout cet héritage ne s'est pas volatilisé sans laisser quelques traces: on peut évoquer sous cette rubrique l'attachement vivace de beaucoup de communautés rurales contemporaines au caractère inaliénable de la terre : que celle-ci puisse être achetée et vendue comme marchandise est à leurs yeux profondément matière à scandale. On peut également signaler leur défiance à l'égard du marché et leur prédilection pour la production de valeurs d'usage, au premier chef de biens de subsistance.

Enfin, il faudrait se garder de peindre un tableau trop idyllique. Le respect de la nature n'a pas été la règle en tous temps ni en tous lieux, et l'histoire a enregistré plusieurs cas de "dérapage ", affectant à vrai dire des sociétés qui n'étaient plus tout à fait primitives. On trouvera dans le remarquable ouvrage de Jared Diamond (2006), l'analyse de quelques-uns de ces cas: habitants de l'île de Pâques, Anasazi du Nouveau-Mexique, Maya du Yucatan. L'essentiel est que chaque fois la catastrophe finale a été provoquée par une utilisation excessive des ressources disponibles - eau, végétation, sol. C'est donc bien une attitude d'arrogance et d'irrespect qui a été sanctionnée, et on peut dire qu'ici aussi l'exception confirme la règle.

Qu'en est-il à présent de la diversité des cultures ? Assurément, nous ne disposons que depuis une date très récente d'un inventaire, même grossier, des cultures du monde, et nous sommes donc incapables de savoir comment, dans la longue durée de l'histoire, a évolué la diversité des cultures. Nous avons pris pour la première fois la mesure de cette diversité à l'âge des Grandes Découvertes, c'est-à-dire au XV et au XVI ${ }^{\mathrm{e}}$ siècle. S'était-elle auparavant accrue ? Était-elle restée étale? Impossible de trancher. Toutefois, nous avons de sérieuses raisons de penser qu'au moins dans le monde des sociétés dites primitives elle était demeurée constante ou avait peut-être même augmenté sous l'effet des scissions et des migrations. 
La première de ces raisons est la faible densité du peuplement; grâce à elle, chaque société dispose de l'espace qui lui est nécessaire, et elle peut toujours partir ailleurs si elle s'estime menacée ; en outre, elle est en mesure de s'isoler et, sans s'interdire le contact et l'échange avec d'autres, de conserver une sorte de "quant à soi » nécessaire au maintien de l'identité :

"Pour développer des différences, pour que les seuils permettant de distinguer une culture de ses voisines soient suffisamment tranchés, les conditions sont grosso modo les mêmes que celles qui favorisent la différenciation biologique entre les populations: isolement relatif pendant un temps prolongé, échanges limités, qu’ils soient de caractère culturel ou génétique. Au degré près, les barrières culturelles sont de même nature que les barrières biologiques" (Lévi-Strauss 1983: 39).

Nouvelle preuve - soit dit en passant - de l'appartenance de l'homme à la nature! Bref, une densité de peuplement réduite et l'abondance de l'espace permettent à chaque communauté de garder ses distances et à la diversité de se maintenir égale à elle-même.

La seconde raison est que, dans le monde des sociétés dites primitives, il n'y a pas d'enjeu pour justifier la coalescence ou la fusion des groupes humains. On le sait : beaucoup de sociétés désignent leurs membres d'un mot qui signifie "les hommes"; elles leur réservent donc la pleine essence humaine; du coup, elles ne songent pas à s'ouvrir ou à absorber d'autres collectivités qui ne partagent pas complètement cette essence. Par ailleurs, des concentrations excessives obligeraient les communautés à renoncer à leur mode de vie pour adopter des méthodes plus intensives et plus productives dans la mise en valeur du milieu. Les terres ne peuvent pas être annexées, car, nous l'avons vu, elles sont la propriété des dieux, et, par délégation, des hommes qui ont les premiers traité avec eux. Enfin, il se peut qu'ici et là d'autres hommes soient réduits en esclavage; mais chaque groupe n'en conserve qu'un petit nombre, autrement dit: c'est son existence même qui pourrait être mise en péril. Bref, les sociétés dites primitives n'ont pas d'intérêt à fusionner, et elles sont souvent tentées de s'ignorer; comme le dit Lévi-Strauss, "cette profonde indifférence aux cultures autres était, à sa manière, une garantie pour elles de pouvoir exister à leur guise et de leur côté" (Ibid.: 26).

À souligner ici la nécessité et les bienfaits d'un certain isolement, LéviStrauss ne se contredit-il pas par rapport à ce qu'il écrivait dans Race et Histoire sur la fécondité des rencontres et des échanges? Il n'en est rien; en effet, pour que l'échange soit fructueux et même tout simplement pour qu'il ait lieu, encore faut-il qu'il y ait des partenaires différents; des êtres qui seraient en tous points identiques n'auraient rien à échanger. Entre une communication favorable aux inventions et au progrès et une non-communication nécessaire au maintien de l'identité, un optimum est à trouver : 
"[...] atteindre un seuil, sans doute le plus profitable aux sociétés humaines, où s’instaure un juste équilibre entre leur unité et leur diversité ; et qui maintient la balance égale entre la communication, favorable aux illuminations réciproques, et l'absence de communication, elle aussi salutaire, puisque les fleurs fragiles de la différence ont besoin de pénombre pour subsister" (Lévi-Strauss 1973 : 300).

Lévi-Strauss reprend cette analyse dans "Race et Culture ", avec des formules qui susciteront d'innombrables débats :

"Toute création véritable implique une certaine surdité à l'appel d'autres valeurs, pouvant aller jusqu'à leur refus, sinon même à leur négation. Car on ne peut à la fois se fondre dans la jouissance de l'autre, s'identifier à lui et se maintenir différent. Pleinement réussie la communication intégrale avec l'autre condamne, à plus ou moins brève échéance, l'originalité de sa et de ma création. Les grandes époques créatrices furent celles où la communication était devenue suffisante pour que des partenaires éloignés se stimulent, sans être cependant assez fréquente et rapide pour que les obstacles, indispensables entre les individus comme entre les groupes, s'amenuisent au point que des échanges trop faciles égalisent et confondent la diversité » (in 1983 : 47-48).

Selon Lévi-Strauss, les sociétés dites primitives ont su réaliser cet équilibre, cet optimum, et à qui demanderait où sont leurs créations, il suffirait - sans parler de l'extraordinaire richesse de leur art, de leur musique et de leurs mythes - de rappeler qu'elles ont pu mener à bien la première grande révolution scientifique et technique de l'histoire humaine, à savoir «la révolution néolithique».

Il est clair cependant - et Lévi-Strauss est le premier à le reconnaître que le mouvement de l'histoire va contredire ses deux axiomes et remettre en cause aussi bien l'intégration pleine et entière de l'être humain à la nature, que le maintien de la diversité culturelle. Pour la clarté de l'exposé, nous distinguerons dans ce mouvement deux phases: l'une, préparatoire jusqu'au $X^{\mathrm{e}}$ siècle de notre ère; l'autre, accélérée, à partir de ce même $\mathrm{XVI}^{\mathrm{e}}$ siècle, la période charnière étant marquée à la fois par les Grandes Découvertes et par les commencements de la deuxième révolution scientifique et technique, celle dite «industrielle».

Commençons par la phase préparatoire. À certaines époques et en certains lieux, l'être humain s'est plus ou moins soudainement conçu comme un être à part, radicalement distinct de la nature, relevant par son esprit d'une autre essence qu'elle, et par conséquent situé dans un autre règne, opposé au sien. Écoutons Lévi-Strauss :

"Car n'est-ce pas le mythe de la dignité exclusive de la nature humaine, qui a fait essuyer à la nature elle-même une première mutilation, dont devaient inévitablement s'ensuivre d'autres mutilations? On a commencé par couper l'homme de la nature, et par le constituer en règne souverain ; on a cru ainsi effacer son caractère le plus irrécusable, à savoir qu'il est d'abord un être vivant. Et, en restant aveugle à cette propriété commune, on a donné champ libre à tous les abus » (1973: 53). 
Dans cette mutation, ajouterai-je pour ma part, il faut faire place à l'influence exercée par les grands monothéismes ; dans les doctrines monothéistes, en effet, l'homme devient le partenaire privilégié, chéri et protégé d'un Dieu désormais unique, créateur et tout-puissant; en vertu de ce statut particulier, l'homme est habilité, de par la volonté divine, à commander à la nature et à la regarder comme son bien. Rappelons-nous le discours que, dans la Genèse (1, 28-29), Dieu tient à Adam et Ève :

«Dieu les bénit et leur dit : "Soyez féconds, multipliez[-vous], emplissez la terre et soumettez-la ; dominez sur les poissons de la mer, les oiseaux du ciel et tous les animaux qui rampent sur la terre". Dieu dit: "Je vous donne toutes les herbes qui sont sur la surface de la terre et tous les arbres qui ont des fruits portant semence: ce sera votre nourriture" ".

On sait que, peu de temps auparavant (Genèse 2, 19-20), Dieu a confié à Adam le soin de donner un nom à tous les animaux de la Création : peuton imaginer manifestation plus claire de son pouvoir sur eux? Dans le Coran, Dieu indique à maintes reprises qu'il a créé le monde ou tel ou tel de ses éléments "pour l'homme" $(2,21,29$, etc.), mais de façon plus précise, quatre sourates (XVI, 5-16; XXII, 65; XXXI, 20 ; XIV, 12-33) affirment que Dieu a placé le monde "au service de l'homme", ce qui implique bien, me semble-t-il, l'exercice d'une domination sous l'égide et le contrôle de Dieu.

La mutation qui prend alors place revêt plusieurs aspects. Aspect géographique et sociologique, tout d'abord avec la naissance des villes. Pour la première fois, des êtres humains sont arrachés à la vie paysanne et au travail des champs. Certes, il s'agit d'abord de dirigeants religieux et/ou politiques, entourés de serviteurs, d'artisans et de commerçants; on peut penser qu'ils sont investis, sur la société globale, d'une sorte d'hégémonie intellectuelle et morale; or ils reçoivent leur subsistance, non pas directement de leur propre travail, mais par voie de prestations ou d'échanges. Leur lien avec la nature commence donc inévitablement à se distendre. On se rappelle les commentaires désolés que le spectacle des villes indiennes, visitées en 1950, a inspirés à Lévi-Strauss. Or, l'examen des ruines de Mohenjo Daro ou de Harappa lui fait pressentir que la catastrophe est en réalité très ancienne et que ces cités disparues depuis des millénaires préfiguraient la vie urbaine telle que nous l'observons aujourd'hui (Lévi-Strauss 1955 : 128-129 et 133 sq.). Ailleurs, Lévi-Strauss soulignera les risques que celle-ci fait peser sur l'équilibre mental des individus, dès lors que la ville prolifere comme un champignon vénéneux :

"L'homme des villes se trouve alors coupé d'une nature au contact de laquelle, seulement, peuvent se régler et régénérer ses rythmes psychiques et biologiques [...]. Cette ségrégation de l'homme hors du milieu naturel dont, au moral comme au physique 
il fait indissolublement partie, la contrainte, à quoi l'astreignent les formes modernes de la vie urbaine, de vivre presque entièrement dans l'artifice, constituent une menace majeure sur la santé mentale de l'espèce" (1973: 334).

Sur le plan politique et économique, la naissance des villes est largement contemporaine de celle des empires, et les empires s'accompagnent en règle générale de grands travaux. Les fins de ceux-ci peuvent être économiques - ouvrages d'irrigation, routes, canaux, etc. - ou bien militaires, politiques et somptuaires - murailles, forteresses, palais, temples, pyramides. Du coup, pour la première fois, la «face de la terre» est transformée en profondeur, et dans de nombreux sites de l'Ancien comme du Nouveau Monde, nous observons aujourd'hui encore les traces imposantes de ces altérations.

Au cours de cette même phase préparatoire, la diversité des cultures n'est sans doute guère menacée. Toutefois, nous voyons surgir les premières formes de cet universalisme qui finira beaucoup plus tard par se dresser contre elle. Cette éclosion se produit d'abord dans le champ religieux. Dès les temps les plus anciens, le récit biblique de la Tour de Babel nous présente la différenciation linguistique comme un châtiment divin, destiné à punir l'orgueil des hommes (Genèse 11, 5-9). Au V siècle avant notre ère, par-delà les particularismes religieux de l'époque, la prédication bouddhique s'adresse à tous les êtres humains sans distinction. Pour sa part, Alain Badiou (1997) fait remonter aux proclamations de saint Paul la naissance de l'universalisme; on se rappelle la célèbre affirmation de l'Épître aux Galates (3, 28) : "Il n'y a ni juif ni grec, il n'y a ni esclave ni homme libre, il n'y a ni homme ni femme, car tous vous ne faites qu'un dans le Seigneur Jésus ". Sept siècles plus tard, personne n'est exclu de la mission du prophète Mohammed. Dans ces trois cas, cependant, la thèse universaliste concerne uniquement le domaine spirituel et laisse très largement intacte la diversité réelle, sociale, linguistique et culturelle.

Il en va de même pour les premières manifestations de l'universalisme civique ou politique: la cité grecque introduit l'égalité devant la loi (isonomia), mais seuls les citoyens en bénéficient. Par l'édit de Caracalla, la qualité de citoyen romain est étendue à tous les ressortissants de l'empire, mais rien n'est changé quant à leurs conditions réelles d'existence.

Enfin, le développement des échanges de toute nature - commerciaux, mais aussi culturels, intellectuels et religieux - favorise la naissance et l'essor des langues dites "véhiculaires", parlées par des ressortissants de sociétés et de cultures diverses: pour s'en tenir au monde méditerranéen, on citera la koinè grecque, le latin, l'arabe. Mais ici encore, ces langues s'ajoutent aux langues locales sans les détruire. 
Avec le XVI ${ }^{e}$ siècle de notre ère, la mutation change à la fois de nature et de rythme. En ce qui regarde la rupture entre l'être humain et la nature, le fait majeur est évidemment la deuxième révolution scientifique et technique, dite aussi révolution industrielle. Sur le plan géographique, la nature est bouleversée : surfaces cultivées, mines, voies ferrées, ports, barrages, donnent un caractère artificiel à la plupart de nos paysages. Il s'agit d'un véritable processus de domestication. Désormais largement réifiée - comme en témoignent les récents procédés de l'élevage industriel ou l'utilisation massive d'engrais et de pesticides -, la nature est réduite à l'état de machine au service des besoins humains. Corrélativement, la population, de plus en plus nombreuse, se concentre toujours davantage dans les villes. En 1950, un être humain sur trois était un citadin; la proportion est à présent de un sur deux, et elle atteint les $90 \%$ dans les pays industrialisés.

Sur le plan idéologique, la séparation entre l'homme et la nature, et la domination du premier sur la seconde, se traduisent à travers deux courants d'ailleurs associés.

Sur le plan philosophique, nous assistons, à partir de Descartes, à l'essor conjoint d'une philosophie de la raison et d'une philosophie du sujet. Dans Myth and Meaning, Lévi-Strauss montre comment le progrès de la science a engendré l'attribution à l'intellect d'une sorte de monopole sur la fonction de connaissance :

«Le fossé, la véritable séparation entre la science et ce que nous pourrions appeler la pensée mythique est intervenue au XVII et au XVIII siècle. À cette époque, avec Bacon, Descartes, Newton, la science fut obligée de se construire contre les vieilles générations de la pensée mythique et de la pensée mystique ; et il fut admis que la science ne pouvait exister que si elle tournait le dos au monde des sens, au monde que nous voyons, sentons, goûtons et percevons. Le monde sensible fut regardé comme un monde trompeur. Le vrai monde fut celui des propriétés mathématiques que seul l'intellect était en mesure de saisir et qui était entièrement en désaccord avec le faux témoignage des sens " (1978 : 4, ma traduction).

De fait, c'est toute l'ancienne logique des qualités sensibles qui se trouve ici récusée comme illusoire, et reléguée au musée des erreurs.

Dans le même mouvement, Descartes, au moyen du Cogito, pose le sujet, ou encore le moi, comme le lieu ultime de la vérité. Il transforme les animaux en machines, et proclame que l'homme est «maitre et possesseur de la nature». Ses successeurs feront du sujet ou de la conscience non seulement le foyer du sens mais aussi la source unique de toute existence, au moins pour nous. Les dernières résistances de Kant - la thèse d'une chose en soi, indépendante de notre perception, mais inconnaissable - seront levées avec Fichte, Schelling, Hegel et l'avènement de l'idéalisme allemand. 
Pour sa part, Lévi-Strauss se montre très sévère à l'égard des formes contemporaines de la philosophie du sujet: "La phénoménologie ", écritil dans Tristes Tropiques, "me heurtait dans la mesure où elle postule une continuité entre le vécu et le réel. D’accord pour reconnaître que celui-ci enveloppe et explique celui-là, j'avais appris [...] que le passage entre les deux ordres est discontinu ; que pour atteindre le réel, il faut d'abord répudier le vécu, quitte à le réintégrer par la suite dans une synthèse objective dépouillée de toute sentimentalité » (1955 : 50). Quant à l'existentialisme, Lévi-Strauss lui réserve, dans L'Homme nu, les flèches de son ironie la plus acérée, parlant à son propos de «cette entreprise auto-admirative où, non sans jobardise, l'homme contemporain s'enferme en tête-à-tête avec luimême et tombe en extase devant soi " $(1971: 572)$.

Sur le plan moral et politique, le triomphe de la philosophie du sujet s'accompagne tout naturellement de l'exaltation de «l'humanisme»: celui-ci proclame la souveraineté de l'être humain sur la nature, place ledit être au centre du monde, fait de son autoréalisation la fin suprême de l'histoire ; l'être humain est posé comme valeur ultime à laquelle tout, dans l'univers, doit être subordonné ou sacrifié. Les rappels à la modestie, lancés sous des formes différentes, par Copernic, par Darwin et par Freud, les opérations de décentrement auxquelles ont procédé, chacun à sa manière, ces trois savants, sont restés inutiles. À la fin du XIX siècle, notamment au travers des idéologies du progrès, l'homme revendique désormais une royauté sans partage et sans limites.

Quant à la diversité culturelle, elle est attaquée de deux manières. Tout d'abord la conquête coloniale et, de façon plus générale, l'expansion occidentale entraînent la disparition de nombreuses cultures. Les unes meurent à la suite de l'anéantissement des sociétés qui les portaient, que les membres de ces sociétés aient été exterminés ou décimés par voie de massacre, ou qu'ils aient succombé à des épidémies meurtrières venues d'Europe. Indiens de l'Amérique du Nord et du Brésil, Aborigènes d'Australie, Tasmaniens, habitants de l'île de Pâques, populations de la Terre de Feu voient leurs effectifs fondre dans des proportions dramatiques ou s'annihiler entièrement. Là où il n'y a pas extinction, l'oppression et l'exploitation paralysent les sociétés, les étouffent, les désagrègent et ne laissent subsister que des cultures en ruines. Comme le déplore LéviStrauss dans Tristes Tropiques:

"Les sociétés que nous pouvons étudier aujourd'hui [...] ne sont plus que des corps débiles et des formes mutilées, [...] elles ont été foudroyées par ce monstrueux et incompréhensible cataclysme que fut, pour une si large et si innocente fraction de l'humanité, le développement de la civilisation occidentale» (1955 : 149). 
En outre, contrairement à la thèse lénifiante propagée par l'évolutionnisme, ce qui s'est produit, ce n'est nullement qu'une culture se soit détachée et ait en quelque sorte pris de l'avance sur les autres, attardées mais intactes. Comme le dit très bien Lévi-Strauss :

"Les sociétés que nous appelons aujourd'hui "sous-développées" ne sont pas telles de leur propre fait, et on aurait tort de les concevoir comme extérieures au développement occidental ou restées indifférentes devant lui. En vérité, ce sont ces sociétés qui, par leur destruction directe ou indirecte entre le XVI ${ }^{\mathrm{e}}$ et le XIX ${ }^{\mathrm{e}}$ siècle, ont rendu possible le développement du monde occidental. Entre elles et lui existe un rapport de complémentarité » (1973: 368).

L'Occident serait donc bien mal venu de s'enorgueillir de sa réussite historique; comme l'écrit encore Lévi-Strauss, «l'ordre et l'harmonie de l'Occident exigent l'élimination d'une masse prodigieuse de sous-produits maléfiques dont la terre est aujourd'hui infectée » (1955:27).

Un second processus - pour une part engendré par le premier - vient encore aggraver la situation et mettre en péril la diversité des cultures : la croissance démographique. En suivant Lévi-Strauss, nous avons souligné plus haut que, pour s'épanouir, la diversité culturelle avait besoin d'espace et de distance. Or, la croissance démographique augmente la densité de la population : elle réduit la superficie dont chaque société et chaque culture peuvent disposer pour «mener leur vie». C'est donc une des conditions nécessaires de la diversité qui se trouve ainsi mise en péril.

À vrai dire, pour Lévi-Strauss, la croissance démographique menace non seulement la diversité culturelle mais bien la liberté elle-même dans les conditions concrètes de son exercice. En effet, explique Lévi-Strauss :

" [...] la liberté n'est ni une invention juridique, ni un trésor philosophique, propriété chérie de civilisations plus nobles que d'autres parce qu'elles seules sauraient la produire ou la préserver. Elle résulte d'une relation objective entre l'individu et l'espace qu'il occupe, entre le consommateur et les ressources dont il dispose. Que cette relation devienne trop fragile ou trop tendue et c'est la liberté elle-même qui est frappée d'anémie" (ibid.: 349).

Ici Lévi-Strauss nous propose une analyse extrêmement suggestive du système hindou des castes, et de la règle végétarienne qui lui est associée. À ses yeux, le régime des castes est conçu comme un moyen de préserver à la fois l'égalité et la différence entre les hommes, en les répartissant entre des groupes séparés, et en rendant ces groupes incommensurables les uns par rapport aux autres. Quant à la règle végétarienne, ajoute-t-il, elle "s'inspire du même souci que le régime des castes, à savoir d'empêcher les groupes sociaux et les espèces animales d'empiéter les uns sur les autres, de réserver à chacun une liberté qui lui soit propre grâce à la renonciation, par les autres, à l'exercice d'une liberté antagoniste » (ibid.: 150). 
Or, l'expérience a échoué, du fait qu'entre des castes, déclarées d'abord égales parce que et en tant qu'elles sont incommensurables, s'est peu à peu introduite «cette dose perfide d'homogénéité qui permettait la comparaison et donc la création d'une hiérarchie ». La leçon qu'on peut tirer de cet échec est sans appel, et Lévi-Strauss l'énonce sans détours : "En devenant trop nombreuse, et malgré le génie de ses penseurs, une société ne se perpétue qu'en secrétant la servitude " (ibid.).

Enfin, certaines des évolutions idéologiques qui ont été évoquées plus haut au titre de la rupture avec la nature contribuent elles aussi à restreindre la diversité culturelle. Le progrès de la science s'est accompagné, avons-nous dit, du triomphe de la pensée rationnelle. Or la raison est par excellence l'instrument ou l'agent de l'universel: les règles de la logique s'imposent à tous, les vérités mathématiques sont valables pour tous, en tout temps et en tout lieu, etc. Pour la raison, campée sur le terrain de l'universel et du nécessaire, les particularités, les spécificités ne sont plus que des contingences ou des accidents, secondaires au regard des généralités qui sont l'objet et la fin de la science. Qui voudrait soutenir les droits de ces particularités et leur assurer une légitimité se verrait bientôt accuser de relativisme et exclure des rangs de la communauté éclairée.

De la même façon, l'influence grandissante de la philosophie du sujet favorise l'avènement d'un individualisme et d'un égalitarisme abstraits dont on trouvera une formulation sans équivoque dans la Déclaration des droits de l'homme de 1789. Certes, il n'est pas question de nier les effets heureux qu'ont pu produire cet individualisme et cet égalitarisme abstraits: pour m'en tenir à un seul exemple, l'abolition de l'esclavage est, pour une part essentielle, leur ouvre. Mais il est clair que l'individu dont on cherche à imposer le règne, c'est l'individu tel qu'il a été produit par quelques siècles de l'évolution de l'Europe. Or, au nom de l'universalisme, lui seul a désormais droit de cité; il devient référence et modèle exclusif pour toutes les autres sociétés du monde; par rapport à ce modèle, tout écart est sauvagerie, arriération, tribalisme, ou, s'il se produit dans nos sociétés occidentales, communautarisme.

Pour conclure sur ce point, il se trouve que, pour mesurer le recul de la diversité des cultures, nous disposons d'un indicateur relativement précis : je veux parler du phénomène de l'extinction des langues. En effet, la langue est la traduction et comme l'incarnation de la culture. Par exemple, écrivent Daniel Nettle et Suzanne Romaine, «le vocabulaire d'une langue est l'inventaire de tout ce dont une culture parle, et de ce qu'elle a classifié afin de donner un sens au monde et de survivre dans un éco-système local " (2003: 65). De même la syntaxe exprime-t-elle les manières de penser et les modes de raisonnement utilisés dans la société considérée. Au surplus, 
la langue est la première marque de l'identité d'une communauté et le premier support de son unité. C'est dire que, lorsqu'une langue se perd, la culture correspondante la suit de très près dans la mort. Or, les chiffres sont ici sans équivoque: selon les linguistes, environ la moitié des langues connues dans le monde ont disparu depuis les cinq cents dernières années. Pour s'en tenir à deux exemples, l'Australie est passée de 250 langues à quelques unités, l'Amérique du Nord de 300 à 175, dont six seulement sont parlées par plus de dix mille personnes et ont quelque chance de survivre...

Selon l'Atlas 2009 international des langues en péril, sur les 6912 langues parlées aujourd'hui dans le monde, près de la moitié - soit 2500 - sont menacées à brève échéance. Or, la fin d'une langue est un malheur irréparable, puisque, avec elle, c'est toute une vision du monde, une expérience, une mémoire singulières et irremplaçables qui sombrent sans espoir de retour.

Au point où nous en sommes arrivés, nous pouvons esquisser une sorte de bilan d'étape et jeter un regard sur l'avenir.

En ce qui concerne la rupture avec la nature, l'importance prise par les phénomènes de pollution et par le processus du réchauffement climatique indique à l'évidence que nous approchons d'un seuil de non-retour, au-delà duquel les dégâts commis deviendraient irréparables et menaceraient à terme la survie même de l'espèce. Pour sa part, Lévi-Strauss s'intéresse à d'autres effets, plus pervers parce que plus dissimulés. J'en donnerai deux exemples.

En s'en prenant à la nature, les êtres humains ont tout d'abord sapé un rempart qui les défendait, y compris contre eux-mêmes : "En isolant l'homme du reste de la création, l'humanisme occidental l'a privé d'un glacis protecteur. À partir du moment où l'homme ne connaît plus de limites à son pouvoir, il en vient à se détruire lui-même " (Lévi-Strauss 1988 : 225-226). Ainsi les êtres humains ont-ils ouvert la boîte de Pandore dont sont sortis beaucoup des grands drames qui ont marqué l'histoire du monde depuis le XVI ${ }^{\mathrm{e}}$ siècle :

"Jamais mieux qu'au terme des quatre derniers siècles de son histoire, l'homme occidental ne put-il comprendre qu'en s'arrogeant le droit de séparer radicalement l'humanité de l'animalité, en accordant à l'une tout ce qu'il retirait à l'autre, il ouvrait un cycle maudit, et que la même frontière, constamment reculée, servirait à écarter des hommes d'autres hommes, et à revendiquer, au profit de minorités toujours plus restreintes, le privilège d'un humanisme corrompu aussitôt né pour avoir emprunté à l'amour-propre son principe et sa notion" (Lévi-Strauss 1973 : 53).

En particulier, les grands massacres dont le $\mathrm{XX}^{\mathrm{e}}$ siècle a été le témoin sont bien, dans cette perspective, la conséquence du traitement préalablement infligé à la nature. 
De même, à s'enfermer dans le tête-à-tête avec lui-même, l'homme risque de manquer bientôt d'air et de périr par asphyxie. Aux yeux de Lévi-Strauss, le destin de l'art moderne illustre bien ce péril. Il a renoncé à la représentation et s'est du même coup détaché du monde; mais l'artiste est ainsi condamné à ne trouver qu'en lui-même les sources de son inspiration. Or, livrée à elle-même, l'imagination humaine n'a qu'une puissance limitée; ses ressources s'épuisent vite; dès lors, la création va s'appauvrissant et l'art glisse peu à peu vers le formalisme et le style décoratif. Lévi-Strauss est donc fondé à s'interroger :

"Que pourra devenir l'art dans une civilisation qui, coupant l'individu de la nature et le contraignant à vivre dans un milieu fabriqué, dissocie la consommation de la production et vide celle-ci du sentiment créateur? [...] Une société porte son art comme l'arbre ses fleurs, en raison de leur enracinement dans un monde que ni l'un ni l'autre ne prétend faire totalement sien" (ibid.: 332-333).

Quant au recul de la diversité, Lévi-Strauss lui impute deux conséquences majeures. Tout d'abord, il est aussi et par lui-même un recul de la liberté. C'est que, pour Lévi-Strauss, la liberté en général n'existe pas; il n'y a que des libertés qui sont toujours situées, historiques et concrètes, et qui constituent donc autant de particularités. La liberté se ramène en fait à :

«[...] cette multitude de petites appartenances, de menues solidarités qui préservent l'individu d'être broyé par la société globale et celle-ci de se pulvériser en atomes interchangeables et anonymes; qui intègrent chacun dans un genre de vie, un terroir, une tradition, une forme de croyance ou d'incroyance, lesquels ne s'équilibrent pas seulement les uns les autres à la façon des pouvoirs séparés de Montesquieu, mais constituent autant de contre-forces capables de se dresser ensemble contre les abus de la puissance publique. En donnant un fondement rationnel à la liberté, on la condamne à évacuer ce riche contenu et à saper ses propres assises [...]. La liberté réelle est celle des longues habitudes, des préférences, en un mot des usages» (1983:380).

Ce qui est ici en cause, on le voit bien, c'est la volonté d'imposer un modèle unique, laquelle est contraire à l'idée même de liberté, puisque, par définition, la liberté c'est la possibilité de diverger, de s'écarter, de se différencier, ou encore, comme disait Rosa Luxemburg: "La liberté, c'est toujours la liberté de celui qui pense autrement» (1969: 83).

Mais, en second lieu, le recul de la diversité signifie la marche du genre humain vers une civilisation unique et uniforme à travers le monde. LéviStrauss caractérise très bien le mouvement contradictoire dans lequel nous sommes emportés :

"C'est la différence des cultures qui rend leur rencontre féconde. Or ce jeu en commun entraîne leur unification progressive : les bénéfices que les cultures retirent de ces contacts proviennent largement de leurs écarts qualitatifs, mais au cours de ces échanges, ces écarts diminuent jusqu’à s’abolir» (1988: 206). 
Déjà dans Tristes Tropiques, Lévi-Strauss évoquait un processus analogue : "Chaque parole échangée, chaque ligne imprimée établissent une communication entre deux interlocuteurs, rendant étale un niveau qui se caractérisait auparavant par un écart d'information, donc une organisation plus grande» (1955: 448). Dans Race et Histoire, Lévi-Strauss nous présentait les contacts et les échanges comme les facteurs décisifs de l'uniformisation ; voici qu'ils apparaissent aussi et dans le même temps comme les facteurs décisifs de l'uniformisation.

Le processus est-il irrémédiable et irréversible? Sur ce point, on observe dans la pensée de Lévi-Strauss comme une hésitation. Dans quelques-uns de ses textes, il suggère que, lorsque les différences s'effacent dans un certain domaine et sur un certain plan, les sociétés les recréent bien vite dans un autre domaine et sur un autre plan, en sorte, si je puis dire, que la quantité de diversité présente dans le monde demeure constante. Déjà, dans l'analyse des mythes, Lévi-Strauss avait observé que les variations du mythe ont pour effet de "permettre à des sociétés petites mais nombreuses d'affirmer leur originalité respective en exploitant les ressources d'une dialectique d'oppositions et de corrélations dans le cadre d'une commune conception du monde» (1964: 16). Dans bien des cas, la multiplicité des versions d'un même mythe traduit « le besoin qu'ont des peuples rapprochés de s'éprouver à la fois comme semblables et comme différents" (1971 : 278, cf. aussi 1973 : 299). En brodant ainsi sur une trame commune, chacun peut défendre son individualité sans pour autant rompre avec ses voisins. C'est un processus de même nature qui pourrait préserver nos cultures d'aujourd'hui de se confondre dans une morne uniformité :

"La civilisation occidentale, devenant chaque jour plus complexe et s'étendant à l'ensemble de la terre habitée, manifeste peut-être déjà dans son sein ces écarts différentiels que l'anthropologie a pour fonction d'étudier [...]. S'il existe [...] un optimum de diversité où elle voit une condition permanente de l'humanité, on pourra être assuré que les écarts entre les sociétés et entre les groupes ne s'effaceront jamais que pour se reconstituer sur d'autres plans. Qui sait si le conflit des générations que tant de pays vérifient en ce moment dans leur sein n'est pas la rançon qu'ils payent pour l'homogénéisation croissante de leur culture sociale et matérielle» (Lévi-Strauss 2008a [1961] : 63).

Dans un article d'Études rurales intitulé «La leçon de sagesse des vaches folles ", Lévi-Strauss s'amuse à imaginer que la maladie de la vache folle amènera l'humanité à renoncer à l'alimentation carnée et à l'élevage du bétail ; ainsi de vastes espaces seraient rendus à la sauvagerie et de nouveaux modes de vie s’y développeraient. Ainsi, conclut Lévi-Strauss, «au lieu d'aller vers la monotonie, l'évolution de l'humanité accentuerait les contrastes, en créerait même de nouveaux, rétablissant le règne de la diversité» (2008b [2001] : 48). 
Dans d'autres textes, Lévi-Strauss se montre beaucoup plus pessimiste, la marche vers l'uniformité lui semble inéluctable, parce qu'elle n'est que la face sociale et culturelle de cette croissance de l'entropie qui caractérise le devenir de l'univers. On le sait, la notion d'entropie fait référence à la seconde loi de la thermodynamique établie par Carnot et reformulée par Clausius. Tout travail exige le concours d'une source chaude et d'une source froide, mais, dans le cours même de la transformation, se produit une irrémédiable dégradation de l'énergie en chaleur. Cette déperdition répétée à l'infini conduit à la disparition des écarts de température qui rendaient le travail possible, et à un état d'équilibre désigné comme la «mort thermique " de l'univers. Ce qui est vrai dans le domaine de l'énergie l'est aussi dans le domaine de l'information; on l'a vu, l'information suppose l'écart, la différence, le contraste, que précisément la circulation de l'information vient effacer. Dès lors se profile à l'horizon un état d'uniformité généralisée qui pourrait être désigné comme la «mort culturelle» de l'espèce humaine. Qui dit entropie, dit désordre grandissant, désorganisation progressive : n'est-ce pas cela que produit en fait l'histoire humaine? Reportons-nous à la conclusion de Tristes Tropiques:

"[L'homme] apparaît lui-même comme une machine, peut-être plus perfectionnée que les autres, travaillant à la désagrégation d'un ordre originel et précipitant une matière puissamment organisée vers une inertie toujours plus grande et qui sera un jour définitive. Depuis qu'il a commencé à respirer et à se nourrir jusqu'à l'invention des engins atomiques et thermonucléaires, en passant par la découverte du feu - et sauf lorsqu'il se reproduit lui-même -, l'homme n'a rien fait d'autre qu'allégrement dissocier des milliards de structures pour les réduire à un état où elles ne sont plus susceptibles d'intégration. Sans doute a-t-il construit des villes et cultivé des champs; mais quand on y songe, ces objets sont eux-mêmes des machines destinées à produire de l'inertie à un rythme et dans une proportion infiniment plus élevée que la quantité d'organisation qu'ils impliquent " (1955: 447).

Bref, « la civilisation, prise dans son ensemble, peut être décrite comme un mécanisme prodigieusement complexe ", dont la fonction est "de fabriquer ce que les physiciens appellent entropie, c'est-à-dire de l'inertie» (ibid. : 448). Lévi-Strauss retrouve ici les accents de Gobineau auquel il rend très souvent hommage. Conformément à la pensée dominante de son temps, Gobineau, on le sait, partait de la différence des races pour expliquer la différence des cultures. En un premier temps, disait-il, les croisements successifs provoquent la diversification des cultures; mais ensuite la multiplication des métissages, des mélanges et des brassages efface les différences et conduit l'humanité vers un état de magma où tous sont mêlés et confondus dans l'indistinction et dans une irrémédiable médiocrité. À partir de points de départ différents, c'est bien un avenir de ce genre que nous annonce le texte que je viens de citer. 
Faut-il parler de contradiction? Je ne le pense pas. En dernière analyse, Lévi-Strauss conclurait plutôt, me semble-t-il, au conflit permanent de deux tendances contradictoires, l'une vers l'homogénéité, l'autre vers la production incessante de nouvelles distinctions (Lévi-Strauss 1978 : 10). La croissance de l'entropie serait sans doute dominante, mais le combat contre elle ne s'achèverait qu'avec la fin du genre humain lui-même.

Devant de telles perspectives, que faire? Il n'appartient pas au savant de donner des consignes d'action. Tout au plus pouvons-nous tirer des analyses de Lévi-Strauss, sous notre responsabilité, quelques suggestions et quelques conseils.

En ce qui concerne la diversité, non seulement nous devrions favoriser la survie des cultures autres que la civilisation occidentale n'a pas encore détruites, mais il nous faudrait aussi encourager la naissance et l'essor de nouveaux particularismes. Par exemple, nous devrions nous garder de contester, au nom d'un universalisme abstrait, le droit que possèdent chaque société et chaque culture de protéger son individualité. "Race et Culture " nous l'a appris : cette protection exige un minimum d'isolement, un minimum de repli sur soi. Tant que ces attitudes ne se transforment pas en orgueil et en agressivité, elles sont parfaitement respectables et elles méritent notre approbation.

Mais surtout, il nous faudrait nous défaire du faux humanisme hérité de la tradition européenne; en finir, dit Lévi-Strauss, avec "cette espèce d'emprisonnement que l'homme s'inflige chaque jour davantage au sein de sa propre humanité, [...] cette espèce de monde clos où l'homme, en tête-à-tête avec ses œuvres, s'imagine qu'il se suffit à soi-même. Une sorte de prison idéale. Et plutôt morne...» (1973 : 330).

De telles formules ont valu à Lévi-Strauss d'être accusé d'antihumanisme, et, de fait, certains propos semblent aller dans cette direction : il s'agit, dit-il par exemple, "de comprendre l'être par rapport à luimême et non par rapport à moi » (1955: 50). Ou encore : "Le but dernier des sciences humaines n'est pas de constituer l'homme, mais de le dissoudre " (1962 : 326). À mon sens, on devrait plutôt parler d'un humanisme élargi; élargi d'abord aux limites de l'humanité, de manière à prendre en compte toutes les cultures passées et présentes, mais élargi surtout aux limites du monde de la vie. Le nouvel humanisme impliquerait que les «droits de l'homme » soient désormais fondés, non plus sur sa qualité d'être moral mais sur sa qualité d'être vivant (1983 : 376), en sorte que ses droits s'arrêteraient " au moment précis ou leur existence met en cause l'existence d'une autre espèce» (ibid. : 374). Bref, il faudrait nous mettre en état de suivre le magnifique appel par lequel Lévi-Strauss conclut 
L'Origine des manières de table, et qui nous servira, à nous aussi, de conclusion :

«Une formule à laquelle nous avons fait un aussi grand sort que "l'enfer, c'est les autres" ne constitue pas une proposition philosophique, mais un témoignage ethnographique sur une civilisation. Car on nous a habitués dès l'enfance à craindre l'impureté du dehors.

Quand ils proclament au contraire que "l'enfer, c'est nous-mêmes", les peuples sauvages nous donnent une leçon de modestie qu'on voudrait croire que nous sommes encore capables d'entendre. En ce siècle où l'homme s'acharne à détruire d'innombrables formes vivantes, après tant de sociétés dont la richesse et la diversité constituaient de temps immémorial le plus clair de son patrimoine, jamais sans doute il n'a été plus nécessaire de dire, comme font les mythes, qu'un humanisme bien ordonné ne commence pas par soi-même, mais place le monde avant la vie, la vie avant l'homme, le respect des autres êtres avant l'amour-propre; et que même un séjour d'un ou deux millions d'années sur cette terre, puisque de toute façon il connaîtra un terme, ne saurait servir d'excuse à une espèce quelconque, fût-ce la nôtre, de se l'approprier comme une chose et s'y conduire sans pudeur ni discrétion" (1968: 422).

École des hautes études en sciences sociales, Paris

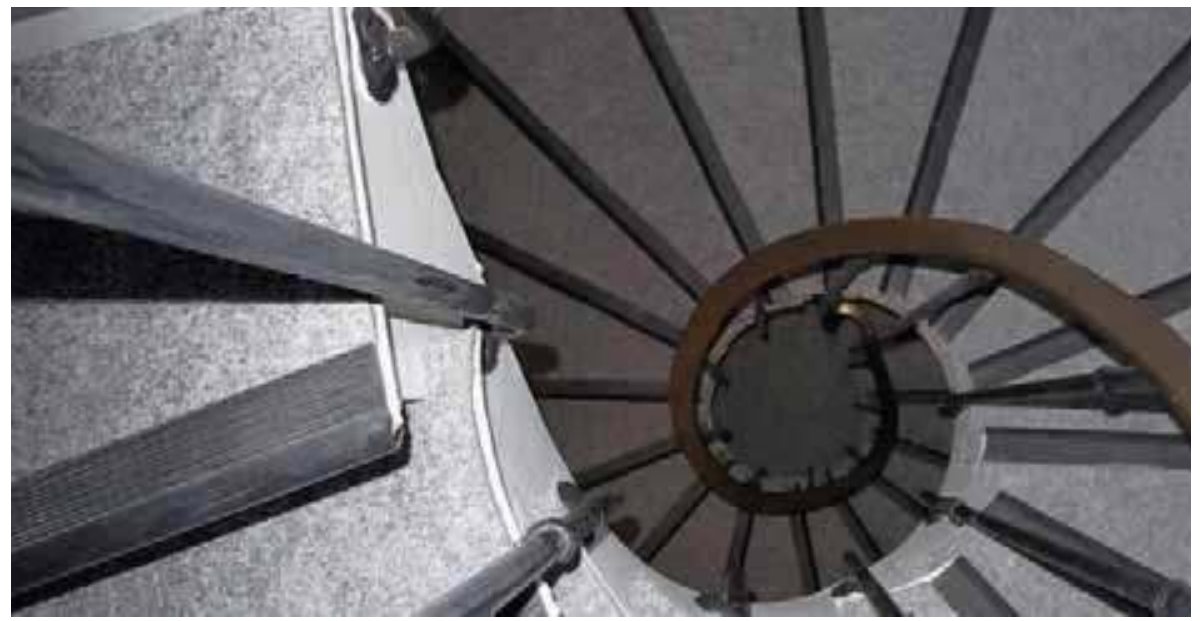

L'escalier en colimaçon conduisant au bureau de Claude Lévi-Strauss

(Laboratoire d'anthropologie sociale, Collège de France, Paris, cl. Jean Jamin, novembre 2009). 


\section{RÉFÉRENCES BIBLIOGRAPHIQUES}

\section{Badiou, Alain}

1997 Saint Paul. La fondation

de l'universalisme. Paris, Presses

universitaires de France.

Diamond, Jared

2006 Collapse. How Societies Choose to Fail or Succeed. London, Penguin Books.

Hamann, Johann G.

2001 "Esthetica in nuce", "Métacritique du purisme de la raison pure" et autres textes.

Trad. de l'allemand par Romain Deygout.

Paris, J. Vrin.

\section{Lévi-Strauss, Claude}

1955 Tristes Tropiques. Paris, Plon.

1962 La Pensée sauvage. Paris, Plon.

1964 Le Cru et le Cuit. Paris, Plon.

1967 Du miel aux cendres. Paris, Plon.

1968 L'Origine des manières de table. Paris, Plon.

1971 L'Homme nu. Paris, Plon.

1973 Anthropologie structurale deux. Paris, Plon.

1978 Myth and Meaning. London, Routledge. [Ma traduction.]
1983 Le Regard éloigné. Paris, Plon.

1988 De près et de loin. Entretien avec Didier Éribon. Paris, Odile Jacob.

2008a [1961] «La crise moderne de l'anthropologie ", Sciences humaines, ${ }^{\circ}$ spécial hors-série 8.

2008b [2001] «La leçon de sagesse des vaches folles ", La Lettre du Collège de France, $\mathrm{n}^{\circ}$ hors-série.

\section{Luxemburg, Rosa}

1969 La Révolution russe, in Euvres, 2 :

Écrits politiques, 1917-1918. Paris, Maspéro.

\section{Nettle, Daniel \& Suzanne Romaine}

2003 Les Langues, ces voix qui seffacent. Menaces sur les langues du monde. Trad. de l'anglais par Marion Guilbault. Paris, Autrement.

Sahlins, Marshall

1968 "La première société d'abondance ", Les Temps Modernes 268 : 641-680.

\section{Tylor, Edward B.}

1958 Primitive Culture. New York, Harper \& Row. 